Décadrages Décadrages

cinéma, à travers champs Cinéma, à travers champs

$7 \mid 2006$

Stephen Dwoskin

\title{
Ultima Thule, un documentaire serti dans la fiction
}

Alain Boillat

\section{(2) OpenEdition}

\section{Journals}

Édition électronique

URL : https://journals.openedition.org/decadrages/474

DOI : $10.4000 /$ decadrages.474

ISSN : 2297-5977

\section{Éditeur}

Association Décadrages

\section{Édition imprimée}

Date de publication : 10 avril 2006

Pagination : 117-118

ISBN : 978-29700582-3-6

ISSN : 2235-7823

Référence électronique

Alain Boillat, « Ultima Thule, un documentaire serti dans la fiction », Décadrages [En ligne], 7 | 2006, mis en ligne le 30 janvier 2014, consulté le 03 avril 2022. URL : http://journals.openedition.org/ decadrages/474; DOI : https://doi.org/10.4000/decadrages.474

Ce document a été généré automatiquement le 3 avril 2022.

(B) Décadrages 


\title{
Ultima Thule, un documentaire serti dans la fiction
}

\author{
Alain Boillat
}

1 Le dernier long métrage du cinéaste Hans-Ulrich Schlumpf, Ultima Thule ${ }^{1}$, résulte d'une tentative quelque peu maladroite mais intéressante de réalisation d'une œuvre hybride où la non-fiction (des vues grandioses tournées en Alaska) est en quelque sorte encapsulée dans une situation-cadre de type fictionnel où un homme d'affaires (Fred, interprété par Stefan Kurt), suite à un grave accident de voiture, tombe dans un comas qui le conduit à voyager dans des espaces mentaux. On pourrait dire que le cinéaste pousse un peu plus loin une formule de fictionnalisation de la non-fiction qu'un producteur comme Jacques Perrin a développée depuis une quinzaine d'années afin de permettre à des documentaires animaliers d'être diffusés dans le cadre de l'institution dominante du cinéma (ses films ne sont pas destinés à la chaîne Planète, mais aux salles de cinéma où ils rencontrent un succès énorme) ${ }^{2}$. Or les expédients inventés par Schlumpf pour enrayer l'autonomisation des longues séquences ressortissant à diverses catégories documentaires (le film de voyage, animalier, scientifique, etc.) ainsi que le montage alterné qui introduit une piste narrative supplémentaire (consacrée à ce qui se passe dans la chambre de l'hôpital lorsque l'épouse du malade lui rend visite) ne font qu'afficher la disparité entre deux pratiques filmiques qui s'opposent à différents niveaux, le descriptif jouant contre le narratif, l'« attraction » des vues spectaculaires contre la narration des moments de mise en scène. La volonté un peu vaine d'inféoder les images documentaires au récit de fiction s'appuie parfois sur la mise en scène d'un regard diégétique aux capacités hypertrophiées. Il s'agit tout d'abord d'un aigle qui emporte avec lui l'«âme » du personnage et motive les vues aériennes; ensuite, d'un télescope astronomique qui introduit une série de photographies cosmiques; enfin, de jumelles qui médiatisent notre accès à différents animaux. Le champ/contrechamp constitue par conséquent le moyen privilégié d'insertion dans le documentaire d'un personnage de fiction, systématiquement assimilé à un sujet percevant. Le montage ne constitue pas l'unique moyen pour rappeler en permanence la présence de l'être de fiction : comme les anges des Ailes du désir (Der Himmel über Berlin, Wim Wenders, 1987), le personnage s'exprime en voix-over et apparaît également en chair et en os 
dans certains paysages. Cette incarnation s'affiche comme un artifice destiné à maintenir la dimension fictionnelle, d'autant plus que le personnage se trouve parfois affecté par les conditions climatiques de son environnement, parfois non, et que l'acteur semble ne pas trop savoir pour quel jeu il doit opter. La narration s'effectue alors exclusivement par le biais du texte over qui nous conte à la première personne le passé du personnage, certaines expressions conduisant à métaphoriser la représentation visuelle simultanée dans une démarche qui se veut "poétique » (par exemple lorsque des gouttes de rosée font écho au mot «larmes»). Le retour aux origines de la vie sur la terre, suggérées par les étendues glacées et totalement inhabitées, occasionne une remise en question de la part du « héros » de son mode de vie, thématique récurrente dans les films suisses depuis Charles mort ou vif (Alain Tanner, 1969). Le courtier en bourse se souvient en effet de sa passion d'adolescent pour les sciences naturelles à laquelle il renonça au profit d'une profession plus lucrative. Ce motif tout helvétique de l'aliénation par le milieu économique permet de développer l'identité du personnage et de conférer à ce voyage une dimension quasi mystique. Dès lors, la fin du film ne peut être qu'une renaissance. A nouveau, le souci de visualiser ce qui est dit, c'est-à-dire de rendre concret un univers mental peut faire sourire (n'est pas Resnais qui veut!), par exemple lorsque Fred retrouve, au milieu de nulle part, le sac à dos de son ancien professeur de géographie qu'il admirait.

2 L'omniprésence du commentaire autobiographique over connait un équivalent dans l'autre piste narrative, puisque l'épouse s'exprime également sur ce mode. $\mathrm{Ce}$ parallélisme nuit autant à la désincarnation de l'esprit du mourant qu'à l'inscription de la situation-cadre dans le quotidien de la vie de l'hôpital, puisque le cinéaste prend la peine de nous montrer le travail qu'effectuent les infirmières autour du patient immobile, c'est-à-dire la part "extérieure " des choses. Lorsqu'on entend la comédienne sangloter over, on se dit que ce procédé n'est peut-être pas le plus adapté à ce genre de situation.

Oscillant entre l'incarnation et le pur espace mental, le documentaire et la fiction, le film de Schlumpf peut sembler bancal, mais il a néanmoins le mérite de proposer une stratégie de conciliation de la pratique documentaire, dominante en Suisse, avec le long métrage de fiction.

Ultima Thule. Eine Reise an den Rand der Welt (2005). Réal. et scénario : H.-U. Schlumpf. Image : P. Corradi, U. Nüesch, J. W. Trapman. Montage : F. Liechti. Son : Dieter Meyer. Interprétation : S. Kurt, B. Auer, P. Frey, Production : Ariane Film AG, Schweizer Fernesehen, Teleclub.

\section{NOTES}

1. Le titre renvoie à " la limite du monde ", ce plus haut point où, dans la conception des Anciens, le voyageur sortait de cette « assiette » qui constitue notre planète. 
2. Perrin a produit consécutivement Le Peuple singe (Gérard Vienne, 1989), Microcosmos, le peuple de l'herbe (Claude Nuridsany et Marie Perennou, 1992) et Le Peuple migrateur (Jacques Perrin, Michel Debats et Jacques Cluzaut, 2001). Notons que lors de sa sortie en Suisse, Microcosmos se trouvait en seconde position du palmarès des entrées en salles, juste après le blockbuster hollywoodien Independence Day. 